\title{
Principle and Outlook of Holography
}

\author{
Yiran Wang ${ }^{1, \text { a }}$ \\ ${ }^{1}$ College of Applied Sciences, Beijing University of Technology, Beijing 100124, China \\ a1311344593@qq.com
}

Keywords: Holography, dynamic holography, digital holography

\begin{abstract}
Holography can display the perspective and depth as a real object, as an ideal three-dimensional display method. As the holography is based on the principle of reestablishing three-dimensional information of an object or a scene by means of optical interference record and diffraction reappearance, the effect of viewing a holographic three-dimensional image is the same as that of viewing a real object or scene. This paper refers to literatures in connection with dynamic holography and digital holography, wherein the dynamic holography is divided into SLM and holographic material, and the current research progress thereof is introduced in this paper; and computer-generated holography is divided into three calculating methods and three processing methods. Meanwhile, this paper introduces the application status of holography and outlooks the future development.
\end{abstract}

\section{Introduction}

Holography has recently become one of hotspots in the study of three-dimensional display as a true three-dimension display technology. The current three-dimensional display technology has defect, so that three-dimensional display cannot reach the level of popularized application. Holographic technology can record and reconstruct all information of a real object or scene, including amplitude information and phase information [1]. Therefore, Holography is an ideal method for three-dimensional display.

\section{Working principle of holography}

Holographic technology is to store all information of light wave which is scattered by a surface of a three-dimensional surface in a recording medium by means of an interference method. When a hologram is illuminated by the same visible light again, light wave of the original object can be reconstructed according to the principle of diffraction, as shown in FIG. 1 [1]. The distribution of interference field not only includes amplitude information (which is also called as color information) of an object, but also includes phase information (which is also called as depth information) of the object. To record the distribution of interference field by means of photosensitive material can achieve the purpose of recording information of the light wave. Holographic reconstruction is to reconstruct object wave in a holographic medium by means of a light beam in particular conditions so as to form new wave that is completely the same as the original object wave for continuous spreading and thereby to form a three-dimensional image. 


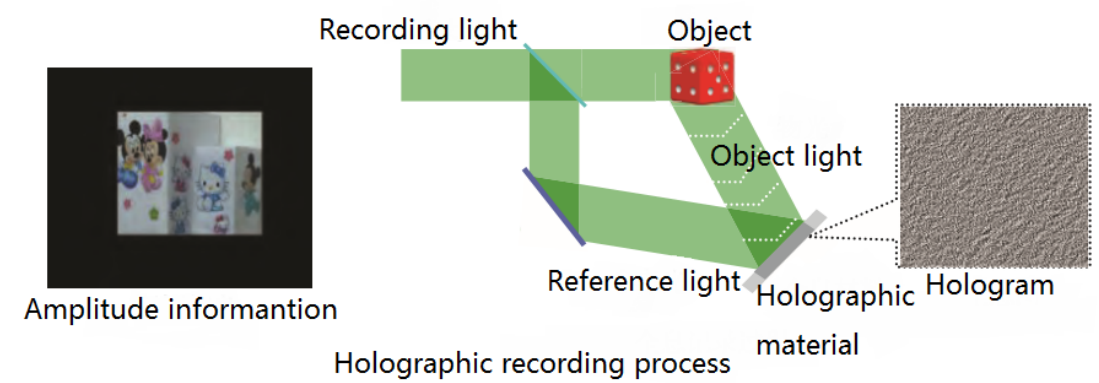

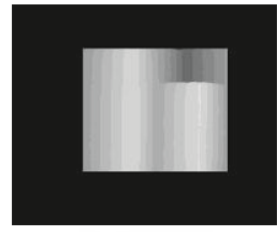

Phase information

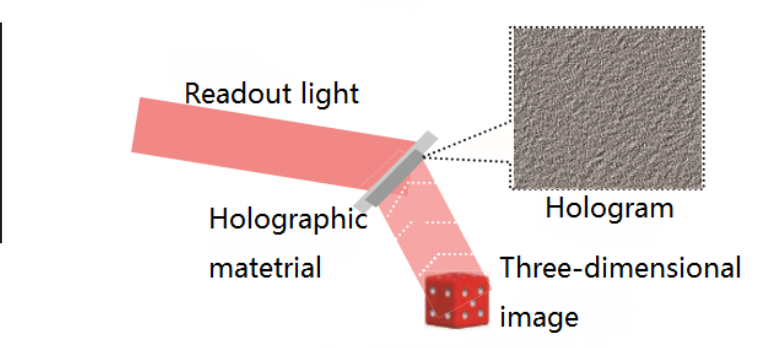

Holographic reconsturction process

FIG. 1 Record and reconstruction of holography [1]

\section{Holographic classification}

Dynamic holography

Dynamic holographic display realized based on spatial light modulator

In 2004, Qinetiq Company employed a method based on a plurality of spatial light modulators to realize holographic color video, with the display dimension of $140 \mathrm{~mm}$ [2]. In 2005, Choi et al systematically combined the integration technology and digital holographic three-dimensional display to improve the visual angle of digital holographic display system [3]. In 2013, Xu et al employed the splicing technology based on a plurality of spatial light modulators to achieve full-parallax holographic three-dimensional display of a larger dimension with the frame rate of $60 \mathrm{~Hz}$. To employ 24 spatial light modulators to realize a holographic three-dimensional video display with the diagonal dimension of 10 inches is as shown in FIG. 2 [4].

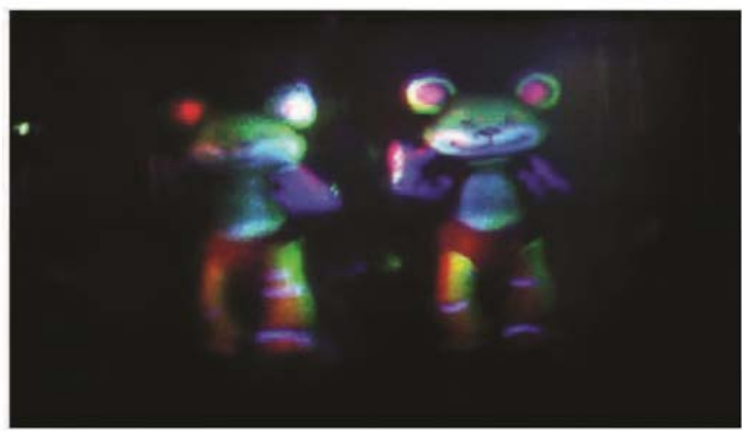

FIG. 2 Full-color and full-parallax holographic reconstruction image [4]

In 2013, Smalley et al developed a holographic video display based on anisotropic leakage patterned modulators, as shown in FIG. 3. Such achievement effectively lowered the cost of digital holographic three-dimensional display [5]. 

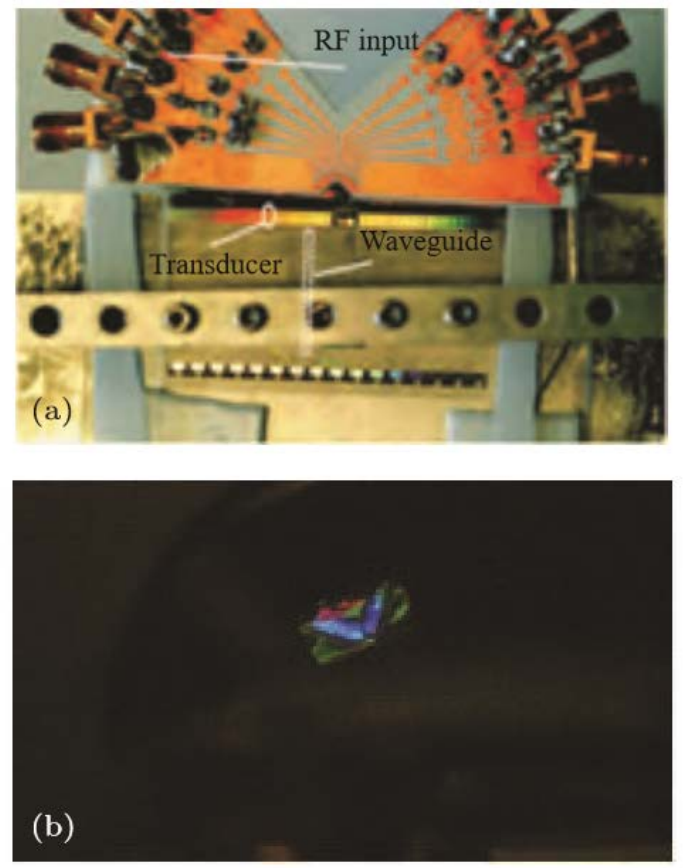

FIG 3 (a) Anisotropic leakage patterned modulators; (b) Color hologram [5]

In 2014, Sasaki et al reported that dynamic full-parallax holographic three-dimensional display with the diagonal dimension of $85 \mathrm{~mm}$, horizontal view angle of 5.6 degrees and refreshing of $20 \mathrm{fps}$ was realized based on 16 spatial light modulators. Sasaki et al employed time division multiplexing technology to realize holographic color display, which was different from the color display which was realized by $\mathrm{Xu}$ et al by means of spatial division multiplexing technology [1].

In order to achieve the purpose of large-dimension holographic display, the holographic display dimension based on the spatial light modulators shall be further increased, and the resolution of three-dimensional display remains to increase. Due to characteristics of the spatial light modulators themselves, the above problems are also pending in the research of the spatial light modulator [1].

Dynamic holographic technology realized based on holographic material

In 2002, Greek scientists, Papa-zoglou et al developed a set of holographic system which employed photorefractive crystal to realize dynamic holographic display, and the reconstructed image was of the dimension of $1 \mathrm{~mm}[6]$. However, it is difficult to grow the photorefractive crystal, which resulted in the failed holographic display of large dimension. In 2010, it was reported in Nature that American Blanche et al achieved a near real-time dynamic holographic display result with the refreshing time of 2 s and display dimension of 4in x 4in in a photorefractive polymer. In 2012, Japanese Kinashi et al reported a quasi-real-time dynamic that the refreshing time in the photorefractive polymer material is $0.2 \mathrm{~s}$, namely the holographic display with the refreshing frequency of $5 \mathrm{~Hz}$. As shown in FIG. 4, Ishii1 et al employed a photochromic material as a holographic medium to realize dynamic holographic refresh. However, the completely erased time for the hologram needed hundreds of milliseconds, and the defects such as a motion trail existed during video refreshing [7]. 


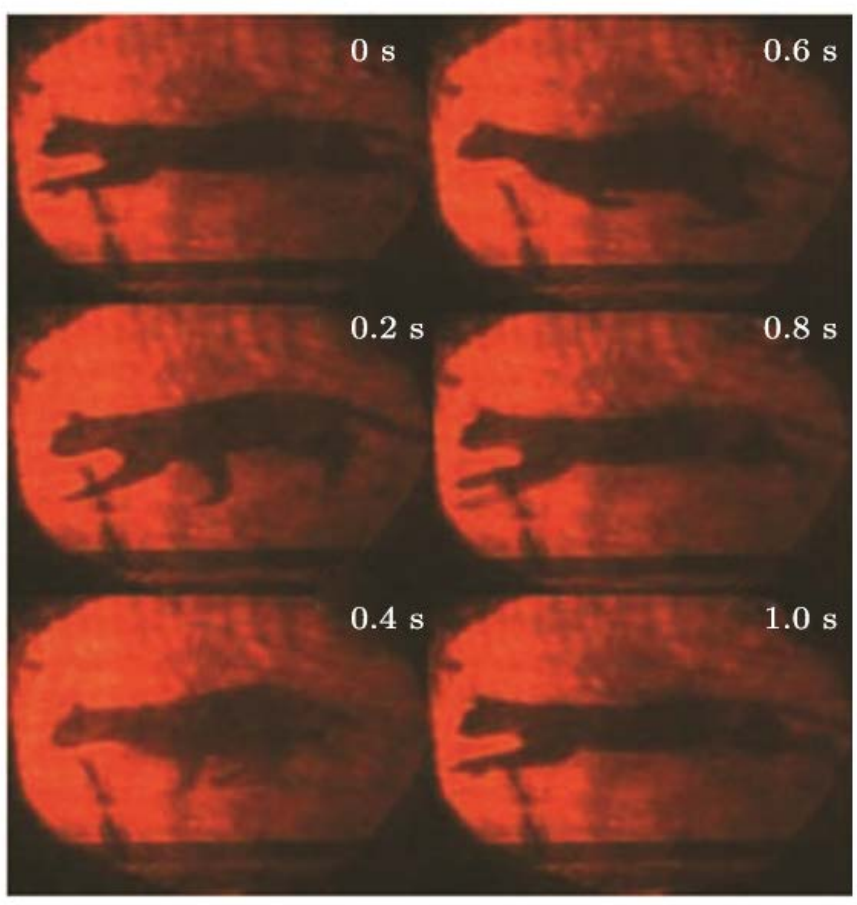

FIG 4 Dynamic hologram image in photorefractive polymer film, with images updated every 0.2s

[7]

\section{Digital holography}

The technology of comprehensively forming a hologram by means of the digitization of object light wave is called as computer-generated hologram or computer-generated holography. The technology of obtaining hologram through direct record or scan by means of a photo electronic imaging device to realize digitalized reconstruction is called as digital holography [8].

Calculating method, including object point scattering method, stereo holography method and chromatography method

Object point scattering method

A three-dimensional object is regarded as a set consisting of a finite number of object points, and each object point is regarded as a point light source. Use a computer to simulate optical propagation of the point light source in a limited direction to obtain the complex amplitude distribution of the point in a holographic plane; and superpose complex amplitudes of all object points to obtain the information distribution of a wave surface of an object in the holographic plane; and therefore a 3D hologram can be obtained by encoding [9].

Stereo holography method

Use a computer or camera to obtain two-dimensional projection images of a three-dimensional object from many angles, and encode the two-dimensional images to obtain a calculating hologram of the three-dimensional object. The first processing method is to add inclination factors into each projection image, conduct integral calculation, make the integrals as pixel values of corresponding points in the projection image from such angle, and combine the pixel values to obtain the hologram. The second processing method is to respectively transform the two-dimensional projection images from Fourier into two-dimensional spectral matrixes and combine all two-dimensional matrixes into a three-dimensional matrix; a two-dimensional matrix which can indicate three-dimensional characteristics of the object can be extracted by analyzing a functional expression of optical field distribution; and a hologram of the three-dimensional object can be obtained by encoding the two-dimensional matrix [10].

Chromatography method

To image a 3D object in a layered manner along the depth direction and add into reference light after superposing complex amplitudes of Fresnel diffractions of respective layers in a holographic 
plane to prepare a hologram of an all object field. The chromatography method is quite applicable to complex virtual 3D objects that are generated by a computer, wherein the encoding is quite easier to realize, the international information of an object can be observed during the reconstruction, and it is particularly suitable for visualization, medical diagnosis, automobile design and like fields featured with complex data, but it is quite difficult to obtain a layered image for an actual 3D object. The quality of a reconstructed image is affected by the number of layers. The more the number of layers is, the better the quality of the reconstructed image is and the larger the calculating amount is [11].

Processing method: based on image sensor recorded hologram and based on computer-generated hologram

Holographic three-dimensional display system based on image sensor recorded hologram

In a conventional holographic recording light path, use a high-resolution image sensor such as a CCD and the like to replace a holographic photographic plate to record an interference-formed hologram; digitalized hologram recorded by the image sensor can be transmitted into a computer for optimized processing and reconstruction [11]. The system has the following defects that it is quite complicated to set up a Mach-Zehnder interferometer for recording light path, and the requirement for surrounding environment is quite higher; it is time-consuming by using a four-step phase-measuring method to record a generated phase hologram; it is difficult to realize dynamic 3D display, and it is also difficult to realize color display by means of a mono-color He-Ne laser.

Digital holographic 3D display system based on computer-generated hologram, including the following three types:

First: Digital 3D display system based on image synthesizing technology

In 2005, k.Choi et al from Seoul National University of South Korea constructed a full-parallax wide-viewing-angle 3D holographic display system by means of a micro lens array, as shown in FIG. 5. A CCD recorded 2D elemental images of a 3D object by means of the micro lens array at the input end; hologram of the elemental images was calculated by means of a corrected Iterative Fourier Transform Algorithm (IFTA), and was uploaded to a spatial light modulator; and a 3D image was reconstructed by means of image formation of the micro lens array at the output end [3].

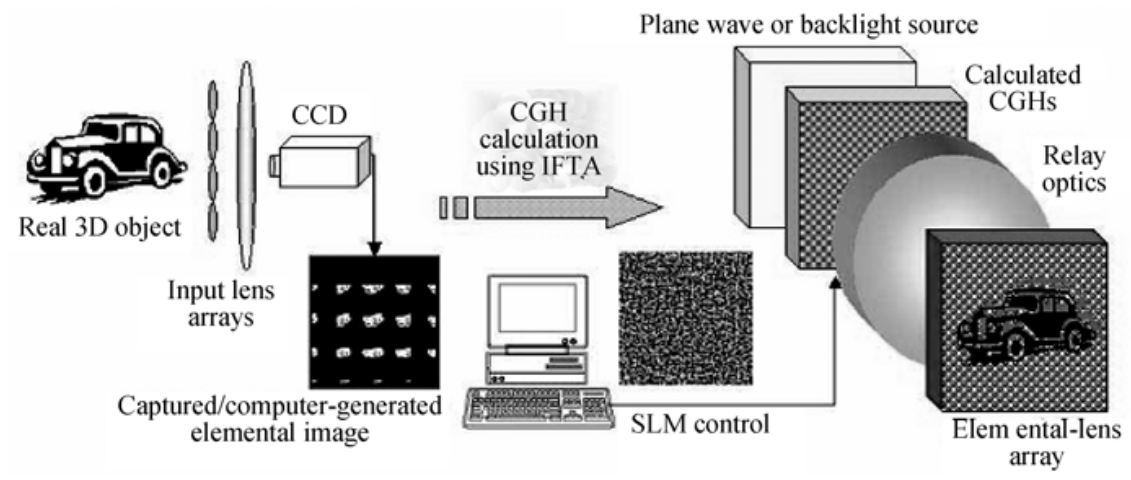

FIG 5 Schematic diagram of full-parallax wide-viewing-angle 3D holographic display system [3]

Second: Holographic 3D display system based on novel recording material

In 2008, P.A.Blanche et al from Arizona State University in the United States prepared a photorefractive crystal and constructed a set of holographic display system available for semi-real-time update, a set of holographic display system available for semi-real-time update; the prepared photorefractive crystal has the advantage of availability for repeated erasion and record; hologram of elemental image of a 3D object is uploaded onto a SLM in turn; SLM-modulated light wave and reference light wave form elemental holograms which are recorded by the photorefractive crystal in turn until a crystal is moved to an optical reconstruction position after finishing recording, and then a 3D image is reconstructed by red light, as shown in FIG. 6 [12]. 


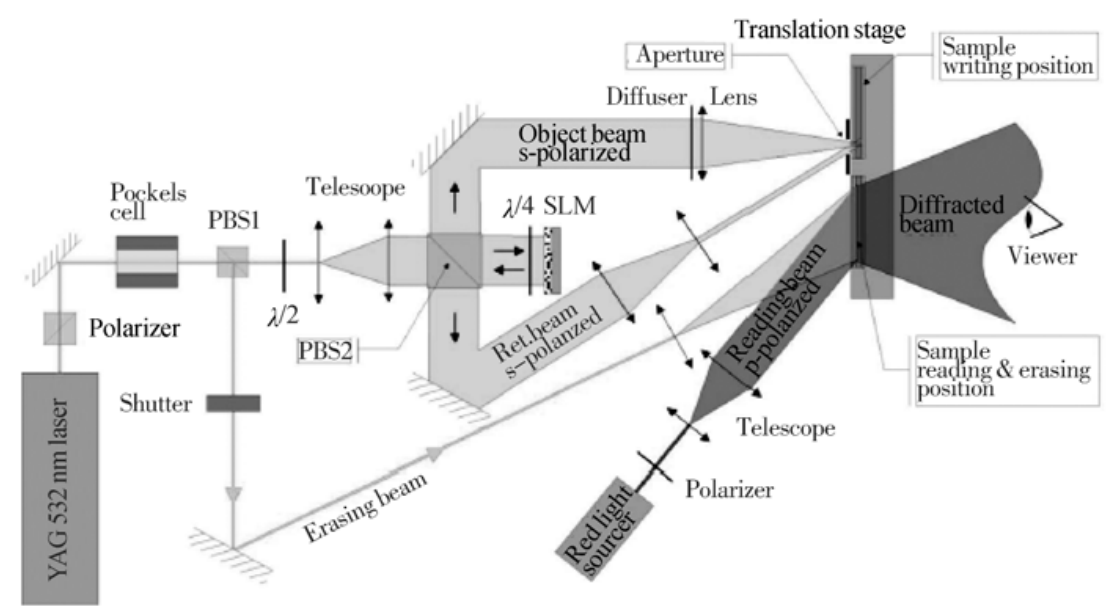

FIG 6 Schematic diagram of 3D display system [12]

Third: Holographic 3D display system based on liquid crystal spatial light modulator or digital micromirror device

A liquid crystal spatial light modulator modulates incident light by means of the electrooptic effect of liquid crystal; a digital micromirror device consists of millions of micro mirrors, and the reflection direction of each micro mirror can be controlled through a voltage signal so as to complete the on/off state of light, so that the modulation to the incident light is realized. In 2002, T.Shimobab et al from Japan employed a reflective liquid crystal display (LCD) panel as a light modulator and light emitting diode (LED) as a reference light source to construct a 3D diagram of an object which was generated by a holographic 3D display system computer by means of an object point scattering method, and the 3D diagram was uploaded to a reflective LCD and was reconstructed by an LED, as shown in FIG. 7. According to the system, it is difficult to obtain a high-resolution reconstructed image as the calculation amount is large due to the employment of the object point scattering method, and moreover the reconstruction effect is affected by shorter coherence length of the LED [13].

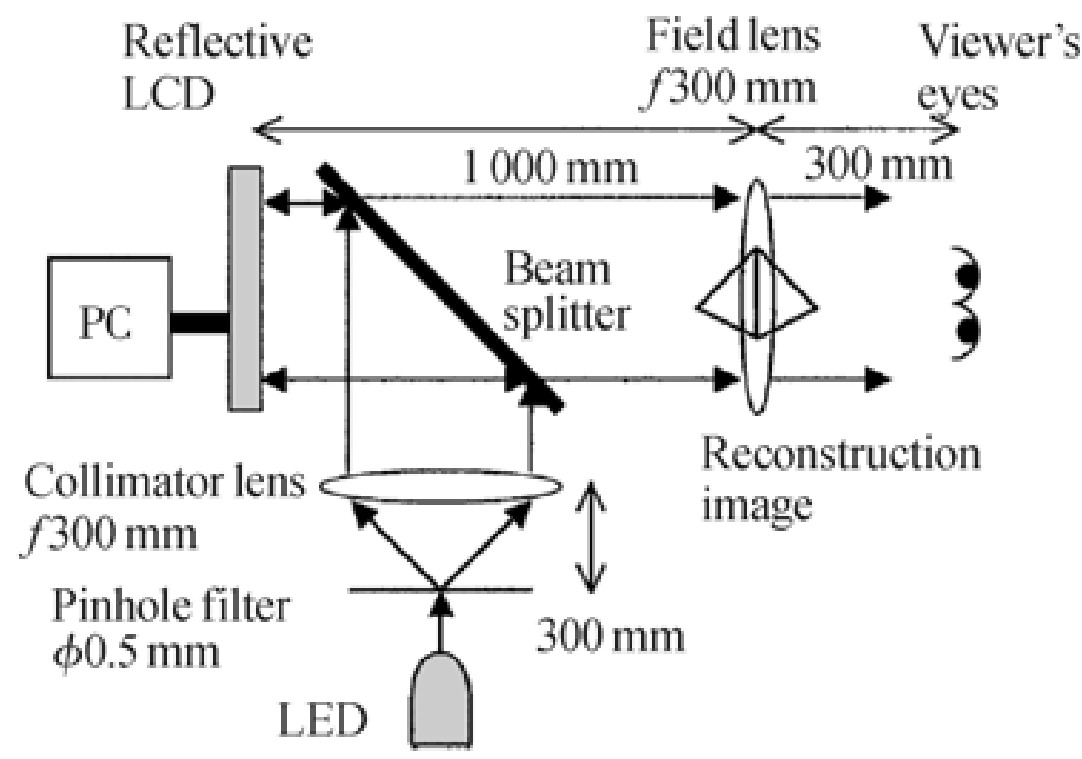

FIG 7 Holographic 3D display system based on LED and LCD [13]

\section{Application field of holography}

The study on digital holography mainly has the following three contents: one is acquisition of digital hologram, the acquisition thereof may also be based on a computer-generated holographic technology, scanning of a photo-electronic imaging device or a method of directly recording optical 
hologram; the second comes the digital reconstruction of reconstructed image of hologram; the third is holographic display, including holographic print and holographic video system [8].

At present, holographic projection has not found wide application. Restricted by technical threshold and capital consumption, holographic projection technology is not widely applied to the living theatres, and it can find more application in large-scale performances at the present stage [14].

Holographic projection technology has broken through the limitation of conventional stage drama plays. It not only can generate stereo phantom, but also can realize the interaction between the phantom and the performer; the appropriate application of holographic projection technology can reach a striking art effect; it solves the problem that some scenes are difficult to realize during the design of a stage, representation forms of the living theatres are enriched; moreover, audiences can interact with performers and the stage in a better manner, so that the audiences can realize the connotation of stories of the living theatres in a more profound manner [15].

Holographic imaging technology has broken through the restriction of conventional sound, light and electricity, which can realize spatial imaging. It is advantageous in color, definition, contrast ratio and the like, and also outstands in spaciousness and perspective. According to the technology, a fantastic world where reality and fiction coexist can be crated [16].

Hologiraphic display mainly makes use of the feature that holography can reconstruct the 3D image of an object. As a holographic photo can present a 3D image which has the same dimension, details and shape as the original object, it is one of promising applications. Such technology can be utilized to reproduce historical culture relics, artistic treasures, holographic portraits, holographic decorations, holographic landscape paintings etc. and to take photos in a super deep field, so that long-distance and close-distance objects can be recorded in one holographic film at the same time. The reconstructed images can be observed in different layers and distances in turn, without being restricted by the depth of field of ordinary photography. Holographic display generally employs the following holography: transmissive \& reflective holography, image plane holography, rainbow holography, true color holography, synthetic holography, embossed holography and like types, wherein all holograms can be reconstructed by white light except for transmissive hologram which needs laser to realize reconstruction, so that a three-dimensional scene can be observed at the natural environment at daytime [17].

Holography is a lensless two-step imaging technology based on the principle of recording an interferogram in anfractuous and differently transparent patterns on a photosensitive film by means of the interference of object light and reference light, which is called as holography (namely holographic photo). Equivalently, a film is prepared into irregular optical gratings, and 3D image is extracted by utilizing diffraction of hologram for proper illuminating light. The later process is called as reconstruction. As natural information storage, hologram can present frozen scenery before human beings again. Due to such unique performance, hologram has found extremely wide applications, such as study on the shock wave during rocket flight, nondestructive test for honeycomb structure of aircraft wings etc. At present, not only laser holography is available, but also white light holography, rainbow holography and full-view rainbow holography have been studied successfully, so that people can see various side faces of scenery. Holographic 3D display is developing towards holographic stereoscopic color television and film [17].

Except for hologram which is generated by light wave, holograms can also be generated by a computer at the present stage. Hologram can find wide application. It can be made into film-type optical elements such as various lenses, optical gratings, filters and the like, which can realize spatial overlapping, are quite compact and light, and are suitable for use in spaceflight. The application of hologram to store data has the advantages of large capacity, easy extraction, fouling resistance and the like. The holography method can be popularized from optical field to other fields. For example, it has witnessed great development in microwave holography and acoustical holography etc., and has successfully applied to industrial medical treatment and like fields. Its applications in seismic wave holography, electronic wave holography and x-ray holography are also in in-depth study [17]. 
To apply holographic technology to anti-counterfeiting field can greatly increase anti-counterfeiting effect. Taking for example a visual reading counterfeiting in the second-generation ID card, viewing the front face of an ID card from a proper angle, there is a sign of Great Wall; while the sign of the Great Wall will change its color as the viewing angle changes [17].

Holography applied to biomedicine can realize quantitative and qualitative analysis on the number of cells of an observed sample. Phase and strength information obtained by means of holographic microscopic imaging technology can be measured accurately. Shapes and characteristics of cells can also be analyzed by means of the technology, so that cancerous cells and damaged cells can be found in advanced by observing cell shapes. It can also be used to record the dynamic process of cell division and infusion, and therefore physical characteristics and variations of cells at respective moments can be fully observed [18].

Holographic display technology can also be applied to cartoon movies, and holograms for 3D display can be synthesized by software by inputting data in a computer. The method has the advantage that light path is not required for record and reproduction; and even a computer can be used to reconstruct a virtue object [19].

\section{Development prospect of holography}

Data compression for digital holograms of 3D object

It is difficult to describe the distribution of object light waves by means of a specific mathematical function due to complicated spatial structure of a 3D object, and 3D computer-generated holography is difficult to calculate due to large amount of 3D information. Therefore, how to decrease data amount of holograms and simplify calculation of the holograms remains an important direction for study [11].

High-resolution wide-viewing-angle video-level dynamic holographic 3D display

Holographic 3D display is a theoretically ideal 3D display technology. To realize high-resolution wide-viewing-angle video-level dynamic holographic 3D display like the real 3D world is a major purpose of researchers [11]. Holographic 3D display can be used to reproduce historical culture relics, artistic treasures, holographic portraits, holographic decorations, holographic landscape paintings etc. and to take photos in a super deep field, so that long-distance and close-distance objects can be recorded in one holographic film at the same time. The reconstructed images can be observed in different layers and distances in turn, without being restricted by the depth of field of ordinary photography [17].

Holographic long-distance reproduction

Online transmission of 3D hologram based on data compression and real-time 3D reproduction of the hologram are also important development directions, such research will realize video connection based on true three-dimensional display [11].

Large-capacity volume holographic storage

In various future high-density storage technologies, people pay attention to holographic optical storage due to its advantages in high storage capacity, high storage density, high information storage redundancy, ultrafast access speed and the like [17]. Spatial multiplexing technology is to divide 2D plane of a recording medium into different areas, and one hologram is separately stored in each area. Volume multiplexing technology is divided into three types: angle multiplexing, phase multiplexing and wavelength multiplexing. Of course, there are many difficulties for the development of holographic optical storage. Firstly, we must look for a storage material having comprehensive advantages in performance, capacity and price, and this is also one of key problems which must be solved during the development of holographic optical storage. Secondly, from the perspective of processing and manufacture, how to process at a lower production cost, in particular in connection with laser, cavity and the alignment of light modulator and detector arrays, remains as a great challenge for engineers. Finally, in order to realize appropriate cost performance, holographic optical storage is difficult to look for its market if the cost is high, and ordinary PC users will not pay high cost for a certain improvement on the performance [17]. 
The application of holographic technology has huge potential, and such novel technology will find applications in various fields such as industry, medicine, national defense and public security and so on, which will lead to various social benefit and economic benefit. As a new-rising subject, holographic technology is still at the stage of prosperous development. With the further development of technology and efforts of scientific and technical personnel, the application of holographic technology is bound to see its more bright future [17].

\section{References}

[1] C. Zeng, H.Y. Gao, J.C. Liu, et al, Latest developments of dynamic holographic three-dimensional display.[J]. Acta Physica Sinica, 2015, 64(12):43-50.

[2] M. Stanley, M. A. Smith, A. P. Smith, et al, 3D electronic holography display system using a 100-megapixel spatial light modulator.[J]. Proceedings of SPIE - The International Society for Optical Engineering, 2004, 5249:297-308.

[3] K. Choi, J. Kim, Y. Lim, et al, Full parallax viewing-angle enhanced computer-generated holographic 3D display system using integral lens array.[J]. Optics Express, 2005, 13(26):10494-502.

[4] X. Xu, X. Liang, Y. Pan, et al, Development of full-color full-parallax digital 3D holographic display system and its prospects.[J]. Proceedings of SPIE - The International Society for Optical Engineering, 2013, 8644(16):2574-2576.

[5] D. E. Smalley, Q. Y. J. Smithwick, Bove Jr V M, Barabas J, S. Jolly, 2013 Nature 498313

[6] D. Papazoglou, M. Loulakis, Holographic read-write projector of video images. [J]. Optics Express, 2002, 10(6):280-5.

[7] K. Kinashi, Y. Wang, S. Tsujimura, et al, Dynamic holographic images using photorefractive composites. [C] Digital Holography and Three-Dimensional Imaging. Optical Society of America, 2012.

[8] H. Wang, Development Status of Digital Holography and Its Application in 3D Display and Detection. [J]. Laser \& Optoelectronics Progress, 2009, 46(8).

[9] Y. H. Wan, J. Zhang, D. L. Pu, et al, Making Three-dimensional Holograms with a Novel Digital Method. [J], Acta Photonica Sinica, 2010, 39(7):1268-1271.

[10] Y. Li, D. Abookasis, J. Rosen, Computer-generated holograms of three-dimensional realistic objects recorded without wave interference. [J]. Applied Optics, 2001, 40(17):2864-70.

[11] J. S. Ma, F. P. Xia, P. Su, et al, Overview on Key Technology and System of Digital Holographic 3D Display. [J]. Optics and Precision Engineering, 2012, 20(5):1141-1152.

[12] P. A. Blanche, S. Tay , R. Voorakaranam, et al, An Updatable Holographic Display for 3D Visualization. [J]. Journal of Display Technology, 2008, 4(4):424-430.

[13] T. Ito, T. Shimobaba, H. Godo, et al, Holographic reconstruction with a 10-micron pixel-pitch reflective liquid-crystal display by use of a light-emitting diode reference light. [J]. Optics Letters, 2002, 27(16):1406-8.

[14]W. X. Yao, Application Study of Digital “Holographic” Projection Technology in Stage Design [J]. China Packaging, 2014(2):28-30.

[15] A. H. Xiao, R. Kong, Influence of Fractal Holographic Technology in Magic and Fantasy Movie Scenes. [J]. Art and Literature for the Masses, 2009(19):112-112.

[16] Z. Hu, Y. Jing, Holographic Imaging Technology in International News [J]. Public Communication of Science and Technology, 2014(22).

[17] J.N. Ni, X.L. Hua, Application and Study on Holographic Technology [J]. ShuZiHua YongHu, 2013, 19(34).

[18] Y.M. Wu, G. Q. Song, Y. P. Feng, et al, Development and Application of Digital Holographic Microscopy in Biomedicine [J]. Imaging Science and Photochemistry, 2016, 34(1):23-29.

[19] N. Farid, M. E. Bahrawi, 3D Display System Based On Digital Holography Application In Making Cartoon Holography [J].Engineering, 2012, 04(7). 